\title{
A generalization of the Coulomb's friction law: from graphene to macroscale
}

\author{
Nicola M. Pugno • Qifang Yin • Xinghua Shi • \\ Rosario Capozza
}

Received: 26 April 2013 / Accepted: 11 July 2013 / Published online: 28 August 2013

(C) Springer Science+Business Media Dordrecht 2013

\begin{abstract}
At the nanoscale, differently to what happens at the macroscale, friction even without an applied normal pressure and spontaneous adhesion take place. In particular, the nanotribology between two layers of graphene, or other two-dimensional nanomaterials (even curved, such as nanotube walls), remains controversial. It is sufficient to say that friction between two graphene layers or nanotube walls is described in the current literature giving as "material property" a constant friction force or a constant friction shear strength, even if such views are obviously mutually exclusive. Is friction dominated by a strength, by a force or by an energy? Coupling elasticity and energy balance we solve this paradox deriving
\end{abstract}

N.M. Pugno $(\varangle)$

Laboratory of Bio-inspired and Graphene Nanomechanics, Department of Civil, Environmental and Mechanical Engineering, University of Trento, Via Mesiano, 77, 38123 Trento, Italy

e-mail: nicola.pugno@unitn.it

N.M. Pugno

Center for Materials and Microsystems, Fondazione Bruno Kessler, Via Sommarive 18, 38123 Povo Trento, Italy

Q. Yin · X. Shi

State Key Laboratory of Nonlinear Mechanics, Institute of Mechanics, Chinese Academy of Sciences, Beijing 100190, China

R. Capozza

Dept. of Physics, Scuola Internazionale Superiore di Studi Avanzati (SISSA), via Bonomea, 265, 34136 Trieste, Italy a generalization of the celebrated Coulomb's friction law, reconciling the two current views. Molecular dynamics simulations on graphene are conducted to verify its validity at the nanoscale whereas statistical simulations confirm its validity even at the macroscale.

Keywords Nanotribology - Graphene - Friction · Molecular dynamics

\section{Introduction}

Graphene, a single-atomic-layer of carbon atoms packed in a honeycomb crystal lattice, has been continuously attracting scientific interest since its first characterization [1] and subsequent experiments showed its two-dimensional lattice stability [2]. Exceptional low-dimensional electronic, thermal, optical and mechanical properties [3-5] make graphene ideal for the development of nanoelectromechanical devices, even foldable and stretchable. Accordingly, graphene could integrate silicon in the next generation of components of integrated circuits and fast transistors, the core of computer processors [6-8] as well as could be used in flexible displays, skin sensors and electronic eye cameras [9-12]. In general, the graphene mechanical behavior is fundamental not only per se but also for designing new applications [13-17], e.g. strain controllable ripples created in suspended graphene are expected to influence its electronic properties [13]. In these systems, the control 
of friction and adhesion and, in general, of nanotribology would crucially enable the designed electronic devices having longer lifecycle. In spite of this, the nanotribology between two layers of graphene, or between other two-dimensional nanomaterials or related systems, remains controversial. Is friction dominated by the friction shear strength, by the friction force or by the dissipated energy? Coupling elasticity and energy balance [18-27] with molecular dynamics (MD) [27-30] and statistical simulations [31-34] we solve in this paper such controversy.

\section{Theory}

We start considering the sliding between a linear elastic continuum plate over a substrate in the presence of adhesion, friction and an external pressure, under a critical pulling force $N_{C}$, Fig. 1. The interaction between plate and substrate is modeled via elastic springs following a classical shear lag model, whereas only locally the validity of the Coulomb's friction law is assumed. We treat the scheme reported in Fig. 1(a) according to elasticity and energy balance, following previous approaches [18-23]; in this paper we introduce friction (i) and we demonstrate that the sliding can be treated as a "delamination" but taking place at a reduced "equivalent surface energy" that is correlated to friction and adhesion (ii). Accordingly, following [18-23], the solution posed by elasticity for the maximum shear stress arising at the interface of the two layers (the stress peak will take place at the end of the element with the higher rigidity, thus is symmetric for our considered identical layers), having Young's modulus $E_{1,2}\left(E_{1,2}=E\right.$ for identical layers), crosssectional areas $A_{1,2}\left(A_{1,2}=A=b h\right.$ for identical layers) and contact length $l=2 c$, in the presence of friction becomes:

$\tau_{\max }=\frac{N \alpha}{b}\left(-C_{1} e^{\alpha c}+C_{2} e^{-\alpha c}\right)-p f$

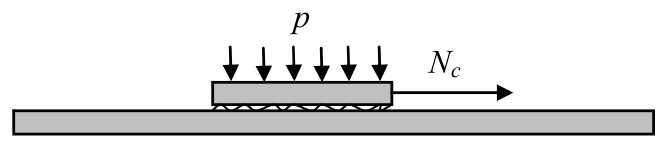

Fig. 1 Schematic shows one linear elastic finite plate sliding over an elastic substrate in the presence of adhesion and friction an of an applied external pressure. The maximal pulling force $N$ is here denoted as "friction force" $N_{C}$

$$
\begin{aligned}
& C_{1}=\frac{e^{-\alpha c}}{e^{-2 \alpha c}-e^{2 \alpha c}}+\beta \frac{e^{\alpha c}-e^{-\alpha c}}{e^{-2 \alpha c}-e^{2 \alpha c}}, \\
& C_{2}=\frac{e^{\alpha c}}{e^{2 \alpha c}-e^{-2 \alpha c}}+\beta \frac{e^{-\alpha c}-e^{\alpha c}}{e^{2 \alpha c}-e^{-2 \alpha c}} \\
& \alpha=\sqrt{\frac{K^{*}\left(E_{1} A_{1}+E_{2} A_{2}\right)}{E_{1} A_{1} E_{2} A_{2}}}, \\
& \beta=\frac{E_{1} A_{1}}{E_{1} A_{1}+E_{2} A_{2}} \geq \frac{1}{2}, \quad K^{*}=\frac{b G_{a}}{h}
\end{aligned}
$$

where $b$ is the nanoribbon width, $G_{a}$ is the shear modulus of the interface between the two layers (thus close to $C_{44}$ of graphite for bilayer graphene), $h$ is the nanoribbon thickness (thus close to $0.34 \mathrm{~nm}$ for bilayer graphene), $p$ is the normal pressure and $f$ is the unknown friction coefficient. The pressure $p$ can be directly linked to the separation distance $h$ between the two layers, assuming a Lennard-Jones (LJ) type potential [24]:

$p(h) \approx \frac{\Psi}{6}\left(\left(\frac{h_{0}}{h}\right)^{10}-\left(\frac{h_{0}}{h}\right)^{4}\right)$

where $h_{0}$ is the equilibrium distance and $\Psi$ is a constant related to the $\mathrm{LJ}$ potential (e.g. for graphite $\Psi=$ $36.5 \mathrm{GPa}[24])$.

Note that posing $\varepsilon=\left(h_{0}-h\right) / h_{0}$ and in the limit of $\varepsilon \rightarrow 0$ we find:

$p \approx \Psi_{\varepsilon}$

Remarkably, from the previous elastic solution two distinct regimes naturally emerge

$$
\begin{aligned}
& \tau_{\max }(\alpha \rightarrow 0)=\frac{N}{l b}-p f \\
& \tau_{\max }(\alpha \rightarrow \infty)=\frac{N \alpha \beta}{b}-p f
\end{aligned}
$$

Accordingly, the corresponding friction force $N_{C}$, arising when $\tau_{\max }=\tau_{C}$, that is the unknown friction strength, is predicted to be:

$N_{C}=\left(\tau_{C}+p f\right) b l, \quad$ for $l \ll l_{C}$

$N_{C}=\left(\tau_{C}+p f\right) \sqrt{\frac{b h}{G_{a}} \frac{E_{2} A_{2}}{E_{1} A_{1}}\left(E_{1} A_{1}+E_{2} A_{2}\right)}$,

for $l \gg l_{C}$ 
where:

$l_{C}=\sqrt{\frac{h}{b G_{a}} \frac{E_{2} A_{2}}{E_{1} A_{1}}\left(E_{1} A_{1}+E_{2} A_{2}\right)}$

is a characteristic structural/material length at which the transition between the two regimes takes place. Note that Eqs. (4a)-(4b) could be seen as generalization of the Coulomb's friction law, i.e. $N_{C}=p f b l$.

Note that the solution posed by Eq. (4a) results in a friction force proportional to the contact length, whereas Eq. (4b) yields a constant friction force, independent from the contact length. The underlying mechanisms related to these two different regimes are generated by the strong heterogeneous shear stress distribution and the adhesion. Only uniform shear stress distributions, present only for "short" contact lengths, may lead to a friction force proportional to the contact area and thus to the Coulomb's law (we are here considering an applied edge force; for a uniform distributed applied shear stress its interfacial distribution is expected to be more homogeneous, mitigating the difference with the classical Coulomb's law). Moreover, the presence of adhesion itself always modifies the Coulomb's law; it is sufficient to say that adhesion could even lead to a negative friction coefficient, since pull-out forces can be generated and thus sliding resistance under a negative pressure, i.e. a negative friction coefficient, can take place [25]. The appearance of the friction force dominated regime is a consequence of the existence of stress-concentrations; these disappear only at critical contact lengths below $l_{C}$. These two distinct regimes have generated a misunderstanding in the previous graphene/nanotube and in general nanotribolgy literature, see comments on [26] and discussed references. For example, we cannot indefinitely increase the strength of a nanotube bundle simply increasing the overlapping length between the nanotubes, as proposed in [27], because the force saturates at a characteristic overlapping length $l_{C}$; rather, we have to design bundle also with longer critical overlapping length $l_{C}$, as pointed out in [28].

A completely independent method for the calculation of the friction force imposes the energy balance during the sliding, interpreting it as a "delamination". Following [18-22], we find the identical result reported in Eq. (4b), if we assume an equivalent dissipated energy per unit surface $2 \gamma$ given by:

$2 \gamma=\frac{1}{2} \frac{\left(\tau_{C}+p f\right)^{2} h}{G_{a}}$
Equation (6) demonstrates that sliding can be seen as a delamination at a reduced surface energy imposed by friction and adhesion. The theory is thus selfconsistent; in the next two sections we will verify it with MD and statistical simulations respectively.

\section{Molecular dynamics simulations}

We here consider the sliding between two, finite or infinite, graphene ribbons having the same width and axial orientation, with or without an applied normal pressure, under a pulling force $N$, see Fig. 2 .

To simulate the sliding behavior of graphene bilayer, we select two graphene nanoribbons having same width $(b=8.376 \mathrm{~A})$, as reported in Fig. 2. The lower one is infinite in $x$-direction and kept fixed along $x$-direction. The periodic boundary condition (PBC) is applied along $y$-direction to eliminate the edge effect. The interaction between atoms at the two free edges (in $x$-direction) of upper graphene and the substrate is turned off to eliminate the edge effect. To induce an applied pressure, the upper sheet is initially arranged to a position with interval $h$ smaller than the equilibrium distance $h_{0}$. With fixed $h$, which is achieved by applying PBC along $z$-direction, the right edge of upper sheet is then pulled rightward at constant velocity of $0.1 \mathrm{~A} / \mathrm{ps}$. The pulling force is captured along the pulling process. By adjusting the interval $h$, we change the induced pressure, and accordingly we obtain various pulling forces. The upper sheet with different lengths ( $l=24,48,96,192,384 \mathrm{~nm}$ ) is also tested to study the size-effect. All the simulations are conducted in an NVE ensemble with code LAMMPS [29], and the AIREBO potential [30] is used to describe the interaction of carbon atoms [31].

It is seen that the equilibrium distance (i.e. when $p=0$ ) for the bilayer is $h_{0}=3.34 \mathrm{~A}$ when the LJ parameters are selected as $\sigma=3.4 \mathrm{~A}$ and $\varepsilon=$ $0.00284 \mathrm{eV}$. The pressure is imposed by tuning the equilibrium distance $h$, Fig. 2 . The circles in Fig. 3 are the results of the MD simulations for graphene bilayer, whereas the line is the prediction of Eqs. (2a)(2b). The shear modulus between layered graphene is also tuned by the distance $h$, Fig. 3, triangle. Note that, considering Eqs. (2a)-(2b) with the theoretical value of $\Psi=36.5 \mathrm{GPa}$ for graphite results in good agreement with the MD simulations for bilayer graphene.

The MD calculated friction forces, normalized by the width of graphene $\left(N_{C} / b\right)$, as a function of contact 

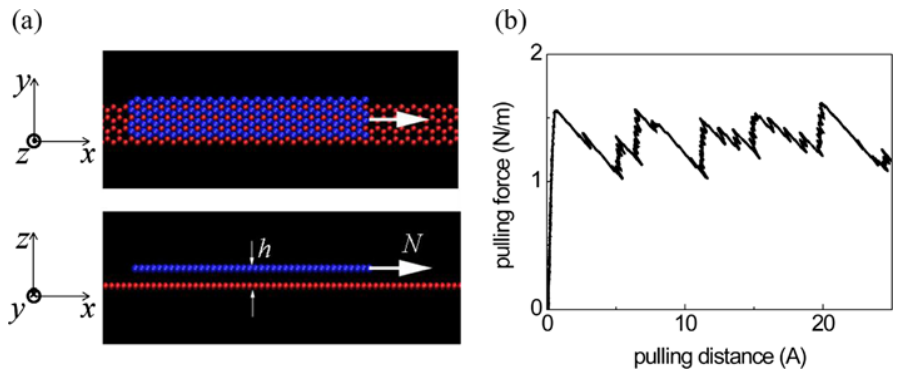

Fig. 2 (a) Schematic shows one finite graphene sheet (upper) sliding on another infinite and fixed (lower). (b) Typical pulling force varies with the pulling distance (here the contact length is $l=48 \mathrm{~A}$ and the pressure is $p=0$; a different pressure can be imposed in the molecular dynamics simulations by tuning $h$ ). The maximal pulling force $N$ is here denoted as "friction force" $N_{C}$

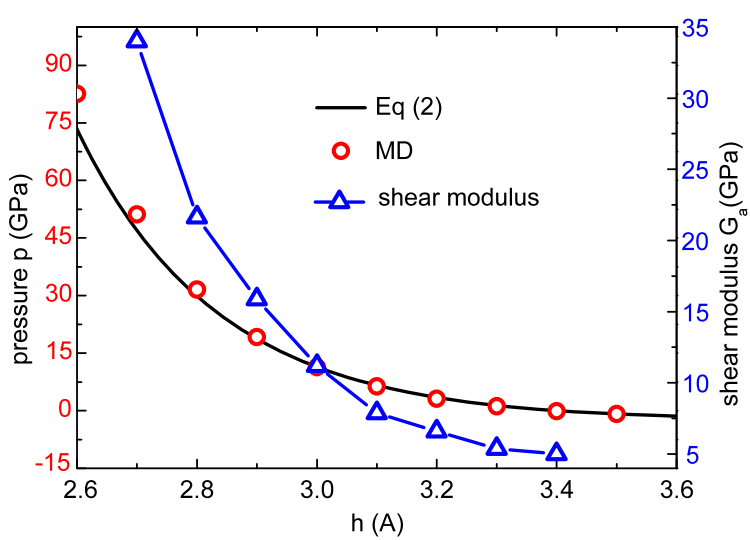

Fig. 3 MD calculations and present theoretical prediction of the variation of pressure, $p$, and shear modulus, $G_{a}$, with the distance between the two graphene sheets, $h$

lengths $l$ and by varying the applied normal pressure are reported in Fig. 4. The circles, squares and triangles are the MD calculations for pressure $p=0,6.5$, and $32 \mathrm{GPa}$, respectively.

The behavior is exactly as theoretically predicted, Eqs. (4a)-(4b), and clearly confirms the existence of the two distinct regimes, strength and then force/energy dominated. In particular, the lines reported in Fig. 4 are the theoretical predictions, Eqs. (4a)-(4b), with best fitted parameters selected as $\tau_{C}=0.08 \mathrm{GPa}$ and $f=0.024$ (from the best fitting reported in Fig. 5). Thus, we can now clearly identify friction strength and friction coefficient of graphene, resulting in an equivalent critical pressure induced by adhesion of $p_{C}=\tau_{C} / f=3.3 \mathrm{GPa}$. The other used parameters are $E_{1}=E_{2}=1109 \mathrm{GPa}, A_{1}=A_{2}=b h$ and the shear modulus is from MD simulations, Fig. 3; thus, from Eq. (4b), we deduce $l_{C}=h \sqrt{2 E / G_{a}}=72 \mathrm{~A}$ as $p=0$ and, from Eq. (6), $\gamma=0.0008 \mathrm{~N} / \mathrm{m}$ that is two orders

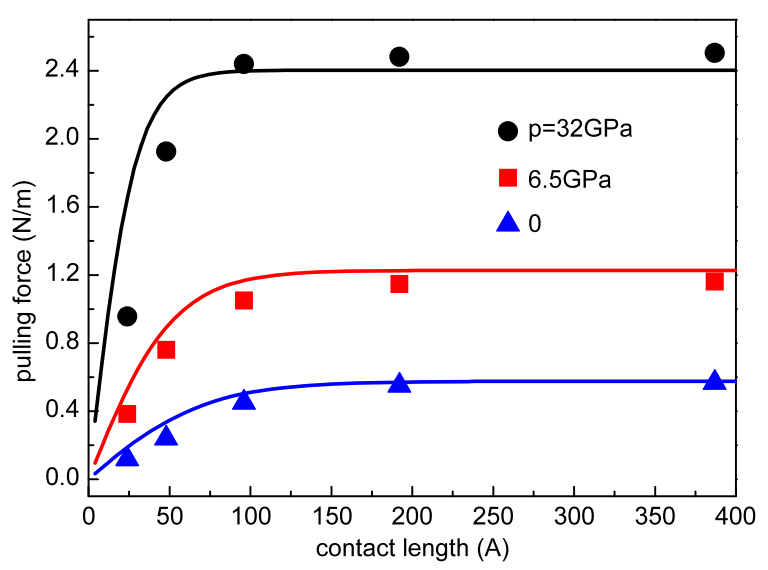

Fig. 4 The friction force normalized by the width of graphene $\left(N_{C} / b\right)$ as a function of contact length $l$. The circles, squares and triangles denote pressure $p=0,6.5$, and $32 \mathrm{GPa}$, respectively. The corresponding lines are from the theoretical prediction, Eqs. (4a)-(4b), with best fitted parameters selected as $\tau_{c}=0.08 \mathrm{GPa}$ and $f=0.024$

of magnitude smaller than the van der Waals adhesion energy, as expected.

In Fig. 5, the friction force per unit width is plotted as a function of the normal pressure $p$. The frictionpressure relation predicted by Eqs. (4a)-(4b) is confirmed by MD simulations (dots in Fig. 5), deriving as a best fit parameters $\tau_{C}=0.08 \mathrm{GPa}$ and $f=0.024$, the same used in Fig. 4.

\section{Statistical simulations}

In order to show the existence of these two frictional regimes also at the macroscale, we consider a macroscopic block (slider) and an immobile rigid substrate with a rough surface, as illustrated in Fig. 6a. The 
elasticity of the slider is taken into account by splitting it into $N_{b}$ rigid and identical blocks with mass $m_{b}=0.164 \mathrm{Kg}$, length $l_{b}=1.6 \cdot 10^{-2} \mathrm{~m}$ and crosssectional area $A_{S}=8.7 \cdot 10^{-3} \mathrm{~m}^{2}$. The blocks are coupled by a set of springs of rigidity $K_{\text {int }}$. The elasticity and length of the slider are then $K_{\mathrm{sl}}=K_{\text {int }} /\left(N_{b}-1\right)$ and $l=l_{b} N_{b}$ respectively. The friction between each block and the substrate is described in terms of $N_{s}$

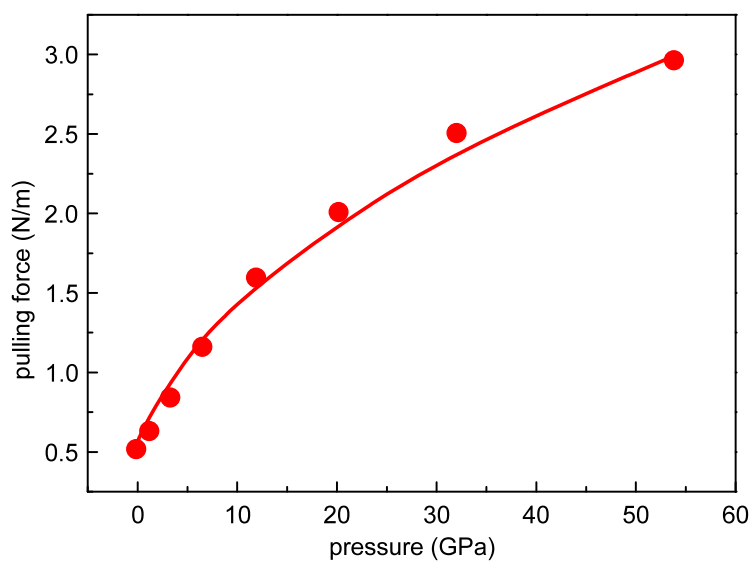

Fig. 5 Friction force $\left(N_{C} / b\right)$ as a function of the normal pressure $p$. The line is a fit of $\frac{N_{c}}{b}=\tau_{c} l_{c}+f l_{c} p$, where $l_{C}=h \sqrt{2 E / G_{a}(p)}$. We deduce $\tau_{c}=0.08 \mathrm{GPa}$ and $f=0.024$ as best fitting parameters elastic interactions representing interfacial asperities $[32,33]$. Each asperity is modeled as a surface spring of elastic constant $k_{i}$ (and average elasticity $k_{s}=\left\langle k_{i}\right\rangle$ ) where $i=1,2 \ldots N_{s}$, and then the shear stiffness $K_{\text {sur }}$ of the entire interface is the sum of the contact stiffnesses $k_{i}$ over all the blocks. When the slider is pulled at constant velocity $V_{d}$, at the beginning the surface springs elongate with the velocity of the corresponding block, opposing a force $f_{i}=k_{i} l_{i}(t)$ against the motion, where $l_{i}(t)$ is the spring length. A contact breaks when $f_{i}$ exceeds a threshold $f_{\mathrm{si}}$ and reattaches in an unstressed state, after a delay time $\tau$. The thresholds $f_{\mathrm{si}}$ are chosen from a Gaussian distribution. The interface stiffness, $K_{\text {sur }}$, and mean rupture threshold force, $f_{s}=\left\langle f_{\mathrm{si}}\right\rangle$, are directly related to the normal pressure supposed uniform along the slider. Artificial vibrations of the blocks are avoided by introducing a viscous damping force with a coefficient $\eta, f_{j}=-m_{b} \dot{x}_{j} \eta$, where $x_{j}$ is the coordinate of the center of mass of the $j$-th block.

In order to simulate the condition of constant pressure along the slider, all parameters are kept constant except for $N_{b}$. By increasing $N_{b}, l$, the shear stiffness $K_{\text {sur }}$ increases, while the elasticity $K_{\text {sl }}$ decreases.

Figure $6 \mathrm{~b}$ shows the friction force as a function of pulling distance obtained when the right edge of a slider of $l=1.6 \mathrm{~m}$ is pulled at constant velocity

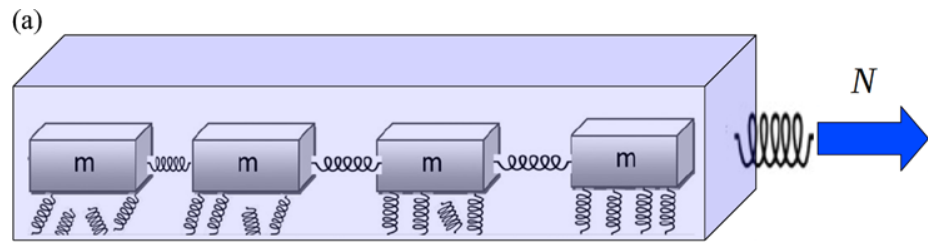

(b)

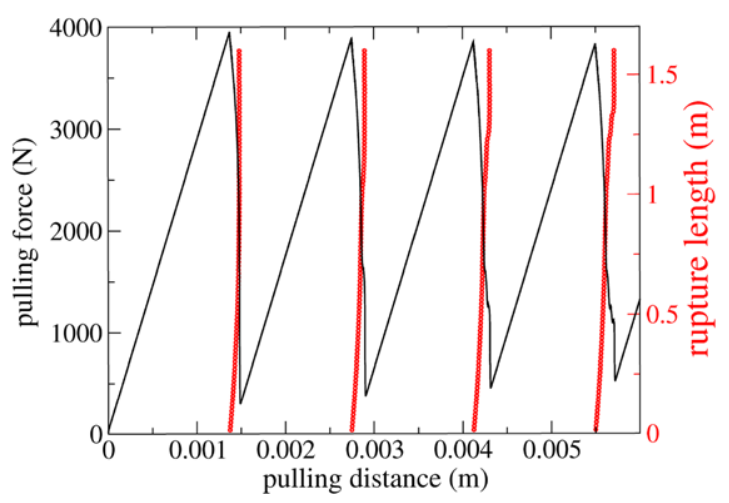

Fig. 6 (a) Schematic sketch of a model setup. (b) The line represents pulling force as a function of pulling distance (displacement of pulled edge) for a slider with length $l=1.6 \mathrm{~m}$. Each slip event is associated to a rupture of the surface springs (nearly vertical lines) propagating from the pulling edge to opposite side 


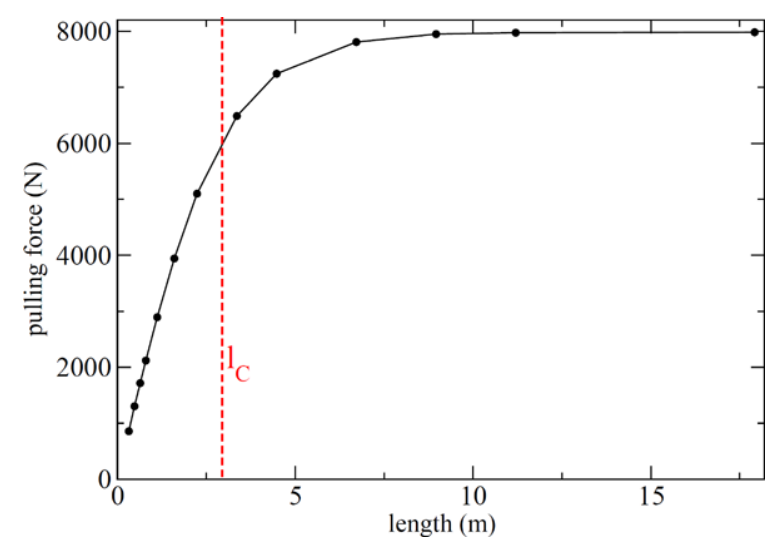

Fig. 7 Friction force $\left(N_{C} / b, b=1\right)$ as a function of slider length. The nearly vertical line indicates the value of $l_{C}$ marking the passage from the linear behavior to the saturation as predicted by Eqs. (4a)-(4b) and MD (see Fig. 4)

$V_{d}=3 \cdot 10^{-4} \mathrm{~m} / \mathrm{s}$. The force grows (stick phase) until surface springs in the vicinity of the loaded edge break and a rupture front propagate [34] from the right to the left edge: at this point the sliding begins and the corresponding force is the friction force. Depending on elasticity of the slider, the dissipation at the interface and the way how the slider is loaded [35], a rupture front can stop before reaching the opposite edge or traverse the entire interface. In our case the sliding is characterized by a single detachment event, as shown by red vertical lines in Fig. $6 b$.

In this model, the shear stress accumulated at the pulling edge decays exponentially along the slider and the corresponding displacement $u(x)$ is described by the equation [35]:

$u(x)=\Delta x_{1} \exp \left[-x /\left(l \sqrt{K_{\mathrm{sl}} / K_{\mathrm{sur}}}\right)\right]$

where $x$ is the position along the slider, $\Delta x_{1}$ is the displacement of the pulling edge. Relation (7) gives rise to a typical stress decay length $l_{C}=l \sqrt{K_{\mathrm{sl}} / K_{\text {sur }}}=$ $h \sqrt{E / G_{a}}$, that corresponds to (5) with the condition $E_{1}=E_{2}=E$ and $A_{1}=A_{2}$. If we consider a slider with $h=0.1 \mathrm{~m}, E=2 \mathrm{GPa}$ and $G_{a}=0.001 \mathrm{GPa}$, $l_{C} \approx 3 \mathrm{~m}$. Note the scaling of the critical length with respect to the previous case of graphene.

Figure 7 shows the behavior of static friction as a function of slider length. The nearly vertical line indicates the value of $l_{C}$. For values of the slider's length $l \ll l_{C}$, the force needed to set the slider in motion grows linearly with $l$, as predicted by Eq. (4a), while for $l \gg l_{C}$ the pulling force saturates to a constant value as predicted by Eq. (4b). These statistical results confirm the validity also at the macroscale of the two friction regimes.

\section{Conclusions}

We have here derived a generalization of the Coulumb's friction law, in the form of $N_{C}=\left(\tau_{C}+p f\right) b l$ for $l \ll l_{C}$ (friction shear strength dominated regime) and $N_{C}=\left(\tau_{C}+p f\right) b l_{C}$ for $l \gg l_{C}$ (friction force/energy dominated regime). Only for $\tau_{C}=0$ and $l \ll l_{C}$ the classical Coulomb's friction law is recovered; however, such a law does not predict a saturation that, according to our model, is present. This has been fully confirmed at the nanoscale by our MD simulations on graphene and at the macroscale by our statistical simulations. Its existence, never clearly observed at the macroscale, perhaps just as a consequence of the large values of $l_{C}$, could have a profound impact in tribology, e.g. in better understanding earthquakes.

Acknowledgements The research related to these results has received funding from the European Research Council: ERC Starting Grant BIHSNAM on "Bio-inspired hierarchical super nanomaterials" (PI NMP) and ERC Proof of Concept REPLICA2 (PI NMP) and by the Graphene Flaghship (NMP). The work is also partially supported by the National Natural Science Foundation of China (NSFC) (Grant No. 11023001), and MD computation is mainly supported by the Supercomputing Center of Chinese Academy of Sciences (SCCAS) and Shanghai Supercomputer Center (SSC).

\section{References}

1. Novoselov KS, Geim AK, Morozov SV, Jiang D, Zhang Y, Dubonos SV, Grigorieva IV, Firsov AA (2004) Science 306:666-669

2. Meyer JC, Germ AK, Katsnelson MI, Novoselov KS, Booth TJ, Roth S (2007) Nature 446:60-63

3. Novoselov KS, Geim AK, Morozov SV, Jiang D, Katsnelson MI, Grigorieva IV, Dubonos SV, Firsov AA (2005) Nature 438:197-200

4. Geim AK, Novoselov KS (2007) Nat Mater 6:183-191

5. Lee C, Wei X, Kysar JW, Hone J (2008) Science 321:385-388

6. Chen J, Ishigami M, Jang C, Hines DR, Fuhrer MS, Williams ED (2007) Adv Mater 19:3623-3627

7. Ponomarenko LA, Schedin F, Katsnelson MI, Yang R, Hill EW, Novoselov KS, Geim AK (2008) Science 320:356-358

8. Lin YM, Dimitrakopoulos C, Jenkins KA, Farmer DB, Chiu HY, Grill A, Avouris P (2010) Science 327:662 
9. Crawford GP (2005) Flexible flat panel display technology. Willey, New York

10. Ko HC, Stoykovich MP, Song JZ, Malyarchuk V, Choi WM, Yu CJ, Geddes JB III, Xiao JL, Wang SD, Huang Y, Rogers JA (2008) Nature 454:748

11. Jin HC, Albelson JR, Erhardt MK, Nuzzo RG (2004) J Vac Sci Technol B 22:2548

12. Lumelsky VJ, Shur MS, Wagner S (2001) IEEE Sens J 1:41

13. Bao W, Miao F, Chen Z, Zhang H, Jiang W, Dames C, Lau CN (2009) Nat Nanotechnol 4:562

14. Cerda E, Mahadevan L (2003) Phys Rev Lett 90:074302

15. Xu Z, Buehler MJ (2010) ACS Nano 4:3869

16. Shi XH, Pugno NM, Gao HJ (2010) J Comput Theor Nanosci 7:517

17. Shi XH, Cheng Y, Pugno NM, Gao HJ (2010) Small 6:739

18. Pugno N (1999) Int J Mater Prod Technol 14:476-487

19. Pugno N, Surace G (2000) Struct Eng Mech 10:125-138

20. Pugno N, Surace G (2001) J Strain Anal 36(1):17-24

21. Pugno N (2001) Int J Mech Control 2:19-27

22. Pugno N, Carpinteri A (2001) Fatigue Fract Eng Mater Struct 25:55-62

23. Pugno N, Carpinteri A (2003) J Appl Mech 70:832-839
24. Zhao YX, Spain IL (1989) Phys Rev B, Condens Matter 40:993-997

25. Deng Z, Smolyanitsky A, Li Q, Feng XQ, Cannara RJ (2012) Nat Mater 11:1032

26. Pugno N (2007) J Mech Behav Biomed Mater 18(4):265-281

27. Vilatela JJ, Elliott JA, Windle AH (2011) ACS Nano 5:1921-1927

28. Pugno N (2010) J Mech Phys Solids 58:1397-1410

29. Plimpton S (1995) J Comput Phys 117:1-19

30. Stuart SJ, Tutein AB, Harrison JA (2000) J Chem Phys 112:6472-6486

31. Mohr M, Maultzsch J, Thomsen C (2007) Phys Rev B 75:153408

32. Braun OM, Barel I, Urbakh M (2009) Phys Rev Lett 103:194301

33. Capozza R, Rubinstein SM, Barel I, Urbakh M, Fineberg J (2011) Phys Rev Lett 107:024301

34. Rubinstein S, Cohen G, Fineberg J (2004) Nature (London) 430:1005

35. Capozza R, Urbakh M (2012) Phys Rev B 86:085430 